\title{
Sphere Wakes at Moderate Reynolds Numbers in a Turbulent Environment
}

\author{
J.-S. Wu* and G. M. Faeth $\dagger$ \\ University of Michigan, Ann Arbor, Michigan 48109
}

\begin{abstract}
The structure of sphere wakes in a turbulent environment was measured over the following test range: sphere Reynolds numbers from 135 to 1560 , ambient turbulence intensities of roughly $4 \%$, ratios of streamwise spatial integral scales to sphere diameters of 11-59, and ratios of Kolmogorov length scales to sphere diameters of 0.080.80. At these conditions, some phenomena observed for sphere wakes at comparable Reynolds numbers in nonturbulent environments were suppressed, like the fast-decaying and self-preserving turbulent wake regions; instead, while the wakes were turbulent, their mean streamwise velocities scaled like self-preserving laminar wakes but with enhanced viscosities due to turbulence. Effective turbulent viscosities were relatively independent of position and ratios of integral length scales and Kolmogorov microscales to sphere diameters; however, they progressively increased with sphere Reynolds numbers. Low and high Reynolds number regimes were observed, separated by a transition regime involving sphere Reynolds numbers in the range from 300 to 600 , which was associated with conditions where effects of vortex shedding were prominent in the temporal power spectra measured in the near-wake region.
\end{abstract}

$C_{d}$
$D$
$d$
$E_{v}(f)$
$f$
$f_{o}$
$\ell$
$e_{K}$
$R e$
$R e_{D}$
$R e_{t}$
$R e_{w}$
$r$
$S t$
$\bar{U}_{t}$
$\bar{U}_{\infty}$
$\bar{u}^{\prime}$
$\bar{u}^{\prime}$
$\bar{v}^{\prime}$
$x$
$\Lambda_{u}$
$v_{t}$
$\tau_{u}$

\author{
Nomenclature \\ $=$ drag coefficient \\ $=$ pipe diameter, $\mathrm{m}$ \\ $=$ sphere diameter, $\mathrm{m}$ \\ = power spectral density of cross-stream velocity \\ fluctuations, $\mathrm{m}^{2} / \mathrm{s}$ \\ $=$ frequency, $s^{-1}$ \\ $=$ vortex shedding frequency, $s^{-1}$ \\ $=$ characteristic wake width, Eq. (3), $\mathrm{m}$ \\ $=$ Kolmogorov length scale, $\mathrm{m}$ \\ $=$ sphere Reynolds number, $d \bar{U}_{\infty} / \mathrm{v}$ \\ = pipe flow Reynolds number, $D \bar{U}_{\infty} / \mathrm{V}$ \\ $=$ turbulence Reynolds number, $d \bar{U}_{\infty} / v_{t}$ \\ = local wake Reynolds number, $\ell \bar{u}_{c} / v_{t}$ \\ $=$ radial position, $\mathrm{m}$ \\ = Strouhal number, $f_{0} d / \vec{U}_{\infty}$ \\ = wake-scaling velocity, Eq. (5), $\mathrm{m} / \mathrm{s}$ \\ $=$ mean relative velocity of sphere, $\mathrm{m} / \mathrm{s}$ \\ $=$ mean streamwise velocity, $\mathrm{m} / \mathrm{s}$ \\ $=\mathrm{rms}$ streamwise velocity fluctuation, $\mathrm{m} / \mathrm{s}$ \\ $=\mathrm{rms}$ cross-stream velocity fluctuation, $\mathrm{m} / \mathrm{s}$ \\ $=$ streamwise distance from center of sphere, $\mathrm{m}$ \\ $=$ streamwise integral length scale, $\mathrm{m}$ \\ $=$ kinematic viscosity, $\mathrm{m}^{2} / \mathrm{s}$ \\ $=$ turbulence kinematic viscosity, $\mathrm{m}^{2} / \mathrm{s}$ \\ $=$ temporal integral scale of streamwise velocity \\ fluctuations, $\mathrm{s}$
}

Subscripts

$c \quad=$ centerline value

$0 \quad=$ virtual origin condition

$\infty \quad=$ ambient condition

\section{Introduction}

$\mathbf{T}$

$\mathrm{HE}$ structure and mixing properties of sphere wakes are important fundamental problems having numerous applications,

Received Dec. 16, 1992; revision received July 24, 1993; accepted for publication Aug. 10, 1993. Copyright (C) 1993 by the American Institute of Aeronautics and Astronautics, Inc. All rights reserved.

${ }^{*}$ Graduate Student Research Assistant, Department of Aerospace Engineering, 3000 FXB Building.

$\dagger$ Professor, Department of Aerospace Engineering, 3000 FXB Building. Fellow AIAA. including the properties of dispersed multiphase flows like sprays, rainstorms, and particle-laden flows. Recent work on turbulence generation by dispersed phases in multiphase flows has highlighted the need for more information about sphere wakes at intermediate sphere Reynolds numbers $(10<R e<1000) .{ }^{1,2}$ This prompted earlier measurements of the structure of sphere wakes at intermediate Reynolds numbers in nonturbulent environments. ${ }^{3}$ The objective of the present investigation was to extend this work to sphere wakes in turbulent environments that are more representative of conditions within practical dispersed multiphase flows.

Past research on the flow near spheres and in sphere wakes in nonturbulent environments is reviewed by $\mathrm{Wu}$ and $\mathrm{Faeth}^{3}$ and the references cited therein. Studies of the near wake of spheres have established the nature of the recirculation zone and the characteristics of vortex shedding. ${ }^{3-12}$ Three wake regions have been identified: a fast-decay wake region, observed only when vortex shedding is present, followed in succession by turbulent and laminar wake regions. ${ }^{3}$ Mean streamwise velocity distributions are selfpreserving in the turbulent and laminar wake regions and scale according to classical similarity theories. ${ }^{13-19}$ The transition between the turbulent and laminar wake regions corresponds to a local wake Reynolds number, $R e_{w} \approx 10$; as the laminar wake region is approached, turbulence intensities along the axis decay proportional to $R e_{w}^{7 / 4}$, which agrees with the scaling predicted for the final turbulence decay period of axisymmetric wakes. ${ }^{20,21}$ Clearly, sphere wakes in nonturbulent environments exhibit a rich variety of flow phenomena; however, the relevance of this behavior to wakes in turbulent environments is questionable. There also have been many studies of the effects of ambient turbulence on drag and interphase heat and mass transfer and the structure of boundary layers along surfaces (see Refs. 22-25 and the references cited therein). However, comparable studies of effects of ambient turbulence on wake properties are limited and do not include consideration of the wakes of axisymmetric objects like spheres.

Based on these observations, the present experimental investigation was undertaken to better understand axisymmetric wakes in turbulent environments. The study involved laser velocimetry measurements of mean velocities and turbulence properties in sphere wakes within the stationary turbulence field near the axis of fully developed turbulent pipe flow. This tactic avoids the complications of streamwise variations of ambient turbulence properties due to the relatively rapid decay of grid-generated turbulence. ${ }^{23}$ The ranges of test variables were as follows: $R e$ from 135 to 1560 , ambient turbulence intensities of roughly $4 \%, \Lambda_{u} / d$ of $11-59$, and 
$\ell_{K} / d$ of $0.08-0.80$. These conditions are representative of dispersed multiphase flows, which typically involve intermediate Reynolds numbers and larger integral scales than the characteristic size of dispersed-phase elements. ${ }^{1-3,26}$

\section{Experimental Methods}

\section{Apparatus}

The apparatus consisted of a turbulent flow of air within a long horizontal pipe, with the test sphere mounted on a traversing system near the axis at the downstream end of the pipe. Wake properties were measured using a rigidly mounted laser velocimeter, whereas pipe flow properties were measured using a traversible laser velocimeter. The pipe flow was produced by a variable speed blower. The blower was followed by a honeycomb flow straightener and four screens to provide a nonswirling uniform flow at the pipe inlet. A smooth PVC pipe having an inside diameter of 300 $\mathrm{mm}$ and a length of $16 \mathrm{~m}$ was used to provide the fully developed turbulent pipe flow. Pipe length-to-diameter ratios at the test location were never less than 52 , which is sufficient to provide fully developed turbulent pipe flow in the region of the wakes. ${ }^{18,19}$

The test spheres were polished plastic balls $( \pm 1 \%$ radius tolerance, sphericity within $1 \%$, and surface roughness less than $0.3 \%$ ) with diameters in the range $1.2-5.6 \mathrm{~mm}$. The spheres were mounted on thin stainless steel wires $(51-\mu \mathrm{m}$ diameter for $d \leq 1.6$ $\mathrm{mm}$ and $127-\mu \mathrm{m}$ diameter for $d \geq 3.0 \mathrm{~mm}$ ), which passed through their centers and were sealed with epoxy. The wires were mounted in tension between two struts passing near the top and bottom of the pipe to prevent sphere vibrations. Tests with no spheres present showed that the disturbances of the wire support system were negligible in comparison with effects of the sphere wakes. The optical system for the wake velocity measurements was rigid; therefore, the spheres were traversed using a stepping motor driven linear positioner in the cross-stream direction (positioning accuracy of $5 \mu \mathrm{m}$ ) and a manual linear positioner in the streamwise direction (positioning accuracy of $500 \mu \mathrm{m}$ ). Alignment of the spheres over the full streamwise traverse was facilitated using a small heliumneon laser mounted permanently with its beam directed along the pipe axis, yielding a maximum uncertainty of the cross-stream position of $1 \mathrm{~mm}$ at $x / d$ of about 100 , with proportionately smaller uncertainties at locations nearer to the sphere.

\section{Instrumentation}

Wake structure measurements involved a dual-beam, forwardscatter, frequency-shifted laser velocimeter with the measuring volume centered on the pipe axis, $30 \mathrm{~mm}$ downstream from its end. The measurements were based on the $514.5 \mathrm{~nm}$ line of an argon-ion laser with an optical power of $500 \mathrm{~mW}$. The sending optics included a $3.75: 1$ beam expander that yielded a measuring volume having a diameter of $50 \mu \mathrm{m}$ and a length of $300 \mu \mathrm{m}$. The flow was seeded with oil drops $(1 \mu \mathrm{m}$ nominal diameter $)$ at the inlet of the blower to yield data rates in the range $0.5-2 \mathrm{kHz}$, depending on the mean velocity of the pipe flow. Velocities were found from the low-pass filtered analog output of a burst counter signal processor. Data were collected using a 12-bit analog/digital converter operating at a frequency comparable to the data rate with the break frequency of the filter set at one-half the sampling rate to control alias signals. Mean and fluctuating velocities were obtained using sampling periods of $120 \mathrm{~s}$, whereas temporal power spectra were measured using sampling periods of $300 \mathrm{~s}$. Experimental uncertainties (95\% confidence) largely were controlled by sampling limitations to yield the following values for $\bar{u} / \bar{U}_{\infty}>0.1$ (and inversely proportional to $\bar{u}$ thereafter): mean velocities, less than $5 \%$, rms velocity fluctuations less than $10 \%$, and temporal power spectra less than $20 \%$. All measurements were repeatable within these ranges over a period of testing of several months.

A traversible dual-beam, forward-scatter, frequency-shifted laser velocimeter was used to measure the structure of the pipe flow, based on the 514.5-nm line of an argon-ion laser and using a fiber-optic cable to transmit the beam to the sending optics. The effective optical power at the measuring volume, accounting for losses in the fiber-optic cable, was $150 \mathrm{~mW}$. The optical arrangement yielded a measuring volume diameter of $100 \mu \mathrm{m}$ and a length of $1200 \mu \mathrm{m}$. Flow seeding, data acquisition, data processing, experimental uncertainties, and repeatability for the pipe flow measurements were the same as the wake structure measurements.

\section{Test Conditions}

Test conditions are summarized in Table 1, with the entries appearing in the order of increasing sphere Reynolds numbers. Mean streamwise velocities at the axis of the pipe flow were in the range $1.56-5.35 \mathrm{~m} / \mathrm{s}$ to avoid problems of natural convection disturbances at very low velocities. Thus, relatively small sphere diameters, $1.2-5.6 \mathrm{~mm}$, were required to consider the intermediate Reynolds number range of interest. This yields blockage ratios less than $0.0035 \%$ due to the presence of the sphere, which is negligible. Pipe flow Reynolds numbers were in the range from $3 \times 10^{4}$ to $1.003 \times 10^{5}$, which yielded streamwise pressure gradients of $0.1-1 \mathrm{~Pa} / \mathrm{m}$. Tests at similar sphere Reynolds numbers but with varying pipe flow Reynolds numbers indicated negligible effects on the flow properties of streamwise pressure gradients in this range.

The ambient flow properties summarized in Table 1 are averages over the region where wake structure measurements were made, e.g., $2 r / D \leq 0.2$. In general, pipe flow properties agreed with

Table 1 Summary of test properties ${ }^{\mathrm{a}}$

\begin{tabular}{|c|c|c|c|c|c|c|c|c|c|c|c|}
\hline$\overline{R e}$ & $d, \mathrm{~mm}^{\mathrm{b}}$ & $\bar{U}_{\infty}, \mathrm{m} / \mathrm{s}^{\mathrm{c}}$ & $R e_{D}^{c}$ & $\bar{u}_{\infty}^{\prime} / \bar{U}_{\infty}, \overline{\%}^{c}$ & $\widehat{\Lambda_{u} / d}$ & $\ell_{K} / d^{\mathrm{c}}$ & $C_{d}^{\mathrm{d}}$ & $x_{0} / d$ & $\overline{\bar{U}}_{t} / \bar{U}_{\infty}$ & $v_{t} / v$ & $R e_{l}$ \\
\hline 135 & 1.2 & 1.82 & 34,100 & 3.9 & 59 & 0.80 & $0.95 / 1.05$ & 1.9 & 1.85 & 2.4 & 56.4 \\
\hline 170 & 1.6 & 1.66 & 31,100 & 4.0 & 45 & 0.63 & $0.86 / 0.86$ & 1.8 & 1.77 & 2.6 & 65.6 \\
\hline 215 & 1.2 & 2.91 & 54,600 & 3.8 & 58 & 0.60 & $0.79 / 0.79$ & 1.5 & 1.50 & 3.5 & 61.0 \\
\hline 240 & 1.6 & 2.40 & 45,000 & 3.8 & 44 & 0.49 & $0.76 / 0.76$ & 1.7 & 1.53 & 3.7 & 64.8 \\
\hline 300 & 3.0 & 1.56 & 30,000 & 4.2 & 24 & 0.27 & $0.70 / 0.63$ & 1.5 & 1.35 & 4.3 & 69.0 \\
\hline 305 & 1.6 & 3.00 & 56,300 & 3.9 & 43 & 0.40 & $0.69 / 0.60$ & 1.1 & 1.13 & 5.0 & 60.5 \\
\hline 315 & 1.2 & 4.25 & 79,700 & 3.8 & 55 & 0.40 & $0.69 / 0.60$ & 1.1 & 1.10 & 5.3 & 59.0 \\
\hline 400 & 1.2 & 5.35 & 100,300 & 3.8 & 53 & 0.35 & $0.63 / 0.63$ & 0.8 & 0.95 & 8.3 & 48.0 \\
\hline 410 & 1.6 & 4.04 & 75,800 & 3.9 & 41 & 0.32 & $0.63 / 0.49$ & 0.9 & 0.87 & 7.3 & 56.3 \\
\hline 415 & 3.0 & 2.19 & 41,100 & 4.0 & 24 & 0.21 & $0.63 / 0.63$ & 1.0 & 0.95 & 8.5 & 48.6 \\
\hline 530 & 1.6 & 5.28 & 99,000 & 3.7 & 40 & 0.27 & $0.58 / 0.58$ & 0.8 & 0.84 & 11.5 & 46.1 \\
\hline 610 & 3.0 & 3.22 & 60,400 & 3.9 & 23 & 0.20 & $0.56 / 0.71$ & 0.8 & 0.80 & 16.8 & 36.3 \\
\hline 610 & 5.6 & 1.82 & 34,100 & 3.9 & 13 & 0.17 & $0.56 / 0.56$ & 0.6 & 0.65 & 17.8 & 34.3 \\
\hline 930 & 3.0 & 4.59 & 86,100 & 4.0 & 21 & 0.15 & $0.50 / 0.64$ & 0.7 & 0.68 & 27.3 & 34.1 \\
\hline 930 & 5.6 & 2.70 & 50,600 & 3.9 & 12 & 0.12 & $0.50 / 0.59$ & 0.5 & 0.52 & 27.9 & 33.3 \\
\hline 1560 & 5.6 & 4.61 & 86,000 & 3.9 & 11 & 0.08 & $0.45 / 0.45$ & 0.5 & 0.49 & 44.3 & 35.2 \\
\hline
\end{tabular}

${ }^{a}$ Sphere mounted near the axis of fully developed turbulent pipe flow in air $\left(\mathrm{V}=16 \mathrm{~mm}^{2} / \mathrm{s}\right)$ within a $300-\mathrm{mm}$ inside diameter smooth pipe. Streamwise pressure gradients in the range $0.1-1 \mathrm{~Pa} / \mathrm{m}$.

${ }^{\mathrm{b}}$ Nylon ball with a $51-\mu \mathrm{m}$ diam mounting wire for $d=1.2$ and $1.6 \mathrm{~mm}$; polycarbonate ball with a $127-\mu \mathrm{m}$ diam mounting wire for $d \geq 3.0 \mathrm{~mm}$

${ }^{c}$ Measured average value over the region $2 r / d \leq 0.2$.

${ }^{\mathrm{d}}$ Value computed from Kürten et al. ${ }^{27}$ / value computed from self-preserving laminar-like mean velocity profiles. 
existing measurements in the literature within experimental uncertainties. ${ }^{29,30}$ Turbulence intensities were not constant due to the present relatively low values of $R e_{D}$, yielding values of $3.7-4.2 \%$ over the test range. Streamwise integral length scales were found using Taylor's hypothesis, $\Lambda_{u}=\bar{U}_{\infty} \tau_{u}$, with $\tau_{u}$ found from measurements of the temporal correlation of streamwise velocity fluctuations. ${ }^{18}$ The resulting measured values of streamwise integral length scales were in the range $64-72 \mathrm{~mm}$, decreasing with increasing $R e_{D}$. Thus, integral length scales generally were an order of magnitude larger than the sphere diameters, $\Lambda_{u} / d=11-59$, which is typical of dispersed phases in turbulent multiphase flows. ${ }^{26}$ Kolmogorov scales were taken to be $\ell_{K}=\Lambda_{u} /\left(\bar{u}_{\infty} \Lambda_{u} / v\right)^{3 / 4}$ from Tennekes and Lumley. ${ }^{17}$ The resulting values were in the range $0.42-1.00 \mathrm{~mm}$, generally decreasing with increasing $R e_{D}$. Thus, Kolmogorov length scales were generally smaller than the sphere diameters, $\ell_{K} / d=0.08-0.80$.

The drag coefficients of the spheres for the various sphere Reynolds numbers were computed in two ways: using the correlation of Kürten et al. ${ }^{27}$ for solid spheres in nonturbulent environments and using the measured mean velocity defect within the wake. The two estimates of $C_{d}$ are in reasonably good agreement in Table 1 , with an average error of $6 \%$ over all of the measurements. The fact that ambient turbulence intensities of roughly $4 \%$ have little effect on the drag coefficient is not surprising for the present relatively low values of $R e$, based on existing measurements in the literature. 22

\section{Wake Similarity}

Remarkably, the present wakes in turbulent environments exhibited extended regions where they behaved like self-preserving laminar wakes with constant viscosities. Effects of turbulence were manifested by enhanced viscosities, in comparison with molecular viscosities, which varied with the sphere Reynolds number. To illustrate these relationships, the scaling of self-preserving laminar wake properties will be summarized in the following.

Classical similarity analysis of self-preserving round laminar wakes with constant viscosities yields the following expression for streamwise velocities ${ }^{19}$ :

$$
\bar{u} / \bar{U}_{\infty}=\left(C_{d} \operatorname{Re}_{t} / 32\right)\left[d /\left(x-x_{o}\right)\right] \exp \left(-r^{2} / 2 \ell^{2}\right)
$$

where

$$
R e_{t}=d \bar{U}_{\infty} / v_{t}
$$

The characteristic wake width $\ell$ in Eq. (2) is given by

$$
\ell / d=\left[2\left(x-x_{o}\right) / d \operatorname{Re}_{t}\right]^{1 / 2}
$$

Here, and in the following, an inertial reference frame has been adopted where the velocities of both the sphere and the wake are relative to the ambient fluid. In addition, the present turbulent flow is considered by replacing the steady velocities of laminar wakes with the mean velocities of the turbulent wakes, $\bar{u}$ and $\bar{U}_{\infty}$, and the laminar kinematic viscosity $v$ by the effective turbulent viscosity $v_{t}$. From Eqs. (1) and (3), the effective local wake Reynolds number for the laminar-like turbulent wake region becomes

$$
R e_{w}=\ell \bar{u}_{c} / v_{t}=\left(C_{d} R e_{t}^{3 / 2} / 16\right) /\left[2\left(x-x_{0}\right) / d\right]^{1 / 2}
$$

Thus, Eqs. (1), (3), and (4) imply $\bar{u} / \bar{U}_{\infty}, \ell / d$, and $R e_{w}$ are proportional to $\left(x-x_{0}\right)^{-1},\left(x-x_{0}\right)^{1 / 2}$ and $\left(x-x_{0}\right)^{-1 / 2}$, respectively, in laminar-like turbulent wakes, as long as $v_{t}$ is independent of position within the wake.

The formulation of Eqs. (1-4) is somewhat inconvenient because $v_{t}$ for laminar-like turbulent wakes is not known a priori, unlike $v$ for laminar wakes. Thus, it was helpful to define a wakescaling velocity $\bar{U}_{t}$ as follows:

$$
\bar{U}_{t} / \bar{U}_{\infty}=C_{d} R e_{t} / 32
$$

Then, substituting Eq. (5) into Eq. (1) yields the following expression for streamwise mean velocities within laminar-like turbulent wakes:

$$
\bar{u} / \bar{U}_{t}=\left[d /\left(x-x_{o}\right)\right] \exp \left(-r^{2} / 2 \ell^{2}\right)
$$

where $\bar{U}_{t}$ is found from the value of $\bar{u}_{c}$ where the extrapolation of the velocity variation in the self-preserving region reaches the intercept, $\left(x-x_{o}\right) / d=1$.

\section{Results and Discussion}

\section{Vortex Shedding}

Similar to sphere wakes in nonturbulent environments, ${ }^{3}$ flow behavior was modified substantially by the presence of vortex shedding from the sphere. Thus, the onset and properties of vortex shedding from the spheres in the present turbulent environment will be considered first.

Conditions for the onset of vortex shedding, and vortex-shedding frequencies thereafter, were found from measurements of temporal power spectra in the near wake, similar to Durão et al. ${ }^{31}$ This involved measurements of the temporal power spectra of streamwise and cross-stream velocity fluctuations at the axis and $x / d=4$, where onset conditions were most easily observed. Although effects of vortex shedding were observed for both velocity components, the following discussion will be limited to the temporal spectra of $\bar{v}_{c}^{\prime}$ which exhibited greater effects of vortex shedding.

Some typical temporal power spectra of cross-stream velocity fluctuations at $r / d=0$ and $x / d=4$ are illustrated in Fig. 1. Results are illustrated for sphere diameters of 1.6 and $3 \mathrm{~mm}$, although findings for other sphere diameters were similar. At low Reynolds numbers when vortex shedding is absent, e.g., at $R e=180, E_{v}(f)$ decays monotonically with increasing frequency. The result of vortex shedding, however, clearly is indicated by a spike in $E_{v}(f)$ for $f d / \bar{U}_{\infty}$ on the order of 0.1 . The magnitude of the spike progressively decreases with increasing $R e$, because effects of turbulence in the near-wake region tend to increase with increasing $R e$ (this will be discussed in more detail in connection with Fig. 7). Thus, at the highest Reynolds number illustrated in Fig. 1, the effect of vortex shedding is limited to a rather small peak in $E_{v}(f)$ near $f d / \vec{U}_{\infty}$ $=0.1$. The behavior seen in Fig. 1 for $E_{v}(f)$ is qualitatively similar to $E_{u}(f)$ and for other positions in the near wake, although the magnitude of the peak and its rate of decay with increasing $R e$ was affected by position.

The onset of vortex shedding, based on the appearance of a peak in $E_{v}(f)$, occurred at $R e$ in the range from 260 to 290 for sphere diameters on the range $1.2-3.0 \mathrm{~mm}$. Thus, $R e$ for the onset of vortex shedding for spheres in the present turbulent environments are not very different from earlier findings for spheres in nonturbulent environments. For example, the onset of vortex shedding in nonturbulent environments is reported at $R e=280$ by Wu and Faeth, ${ }^{3}$ 300 by Magarvey and MacLatchy, 270 by Goldberg and Florsheim $^{6}$ and 300 by Sakamoto and Haniu. ${ }^{11}$

The frequencies of vortex shedding also were similar in nonturbulent and turbulent environments. This is illustrated in Fig. 2, where measurements of vortex-shedding frequencies, represented by the Strouhal number, $S t=f_{o} d / \vec{U}_{\infty}$, are plotted as a function of $R e$. Aside from the present results for spheres in turbulent environments, and those of Marshall and Stanton ${ }^{32}$ for a circular plate in a nonturbulent environment, all the results are for spheres in nonturbulent environments. Kim and Durbin ${ }^{12}$ point out that there are two simultaneous modes of unsteadiness for $R e>800$, which accounts for the double-valued behavior in this region. The high- frequency mode is associated with instability of the separating shear layer and is only observed for measurements near the sphere. The low-frequency mode extends to frequencies at the onset of vortex shedding and is associated with the large-scale instability of the wake; as a result, it is called the vortex-shedding mode. ${ }^{12}$

Present measurements were made near the axis at some distance from the sphere and only the vortex-shedding mode was observed. The results illustrated in Fig. 2 indicate a tendency for $S t$ for vortex 


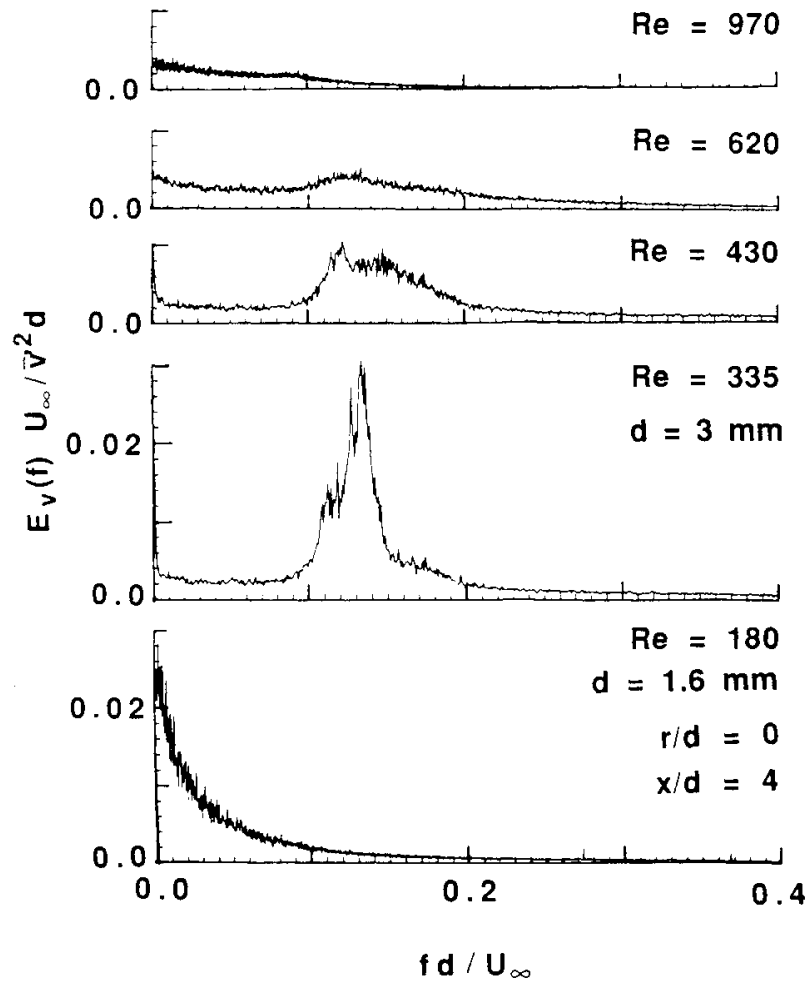

Fig. 1 Temporal power spectra of cross-stream velocity fluctuations in the near-wake region.

shedding to be slightly reduced in turbulent environments in comparison with nonturbulent environments. However, the reduction is not large in comparison to the bandwidth of the spectral peak; see Fig. 1. A somewhat unusual observation was the subharmonic observed near the onset of vortex shedding for the 1.6-mm-diam sphere, although subharmonics have been observed for shear layer instabilities in the past. ${ }^{11,12}$

\section{Mean Velocities}

Measurements of mean velocities along the axis suggested that there was an extended region where the wakes were self-preserving and exhibited laminar-like scaling. Thus, plots of $1 / \bar{u}_{c}$ as a function of $x / d$ were extrapolated to find the virtual origin, whereas the value of $\bar{u}_{c}$ at $\left(x-x_{o}\right) / d=1$ yielded $\bar{U}_{t}$, based on Eq. (6). The resulting values of $x_{0} / d$ and $\bar{U}_{t} / \bar{U}_{\infty}$ are summarized in Table 1. In general, the virtual origins were in the near-wake region, relatively close to the sphere, e.g., $x_{o} / d$ of $0.5-1.9$. The value of $x_{o} / d$ progressively decreased as the $R e$ increased. The ratio, $\bar{U}_{i} / \bar{U}_{\infty}$, was in the range $0.49-1.85$, and also tended to decrease as $R e$ increased.

The variation of mean streamwise velocities along the axis, $\bar{u}_{c} / \bar{U}_{t}$, is illustrated in Fig. 3 for all of the test conditions. The behavior of laminar-like wake scaling according to Eq. (6) is also shown on the plots. The measurements extend from the near-wake region, $\left(x-x_{0}\right) / d$ of $1-2$, to $\left(x-x_{0}\right) / d$ of roughly 100 . The measurements were terminated at large distances from the sphere where mean velocities became small and the experimental uncertainties stated earlier could not be maintained.

The measurements illustrated in Fig. 3 are grouped into three regimes based on behavior in the near-wake region. Measurements in all three regimes, however, clearly exhibit the scaling expected for laminar-like turbulent wakes at $\left(x-x_{0}\right) / d$ in the range of 8 100 . The three regimes involve a low Reynolds number regime, $R e$ $<300$, a high Reynolds number regime, $R e>600$, and a transition regime between these two. The low and high Reynolds number regimes involve similarity in terms of $\bar{u}_{c} / \bar{U}_{t}$ over the full range of $(x$ $\left.-x_{o}\right) / d$; however, values of $\bar{u}_{c} / \bar{U}_{t}$ are lower and higher, respectively, than the extrapolation of laminar-like turbulent wake behavior in the near-wake region $\left(x-x_{o}\right) / d<8$. Behavior in the tran- sition regime represents an evolution between these two limiting conditions, with similarity in terms of $\bar{u}_{c} / \bar{U}_{t}$ much less evident in the near-wake region. The onset of the transition regime at $R e$ of about 300 coincides with conditions where vortex shedding begins. Thus, the changes of near-wake behavior seen in Fig. 3 are caused by effects of vortex shedding, analogous to behavior observed for spheres in nonturbulent environments. ${ }^{3}$

Beyond the near-wake region in Fig. $3,\left(x-x_{0}\right) / d>8$, mean velocities scale reasonably well according to the laminar-like turbulent wake correlation. This implies faster mixing rates as $R e$ increases for the present variation of $\bar{U}_{t} / \bar{U}_{\infty}$ summarized in Table 1 . For example, the values of $\left(x-x_{0}\right) / d$ where $\bar{u}_{c} / \bar{U}_{\infty}$ became small, say $1 \%$, were in the range 24-96, progressively increasing with decreasing $R e$. Values of $R e_{w}$ at this condition were roughly 1.4 for the low Reynolds number regime, and 0.6 for the high Reynolds number regime, with intermediate behavior in the transition regime. Thus, $R e_{w}$ on the order of unity is representative of the end of the wake-mixing process for the present turbulent environment.

Given $\bar{U}_{t}$ and $C_{d}$ for a particular sphere Reynolds number, $R e_{t}$ can be computed from Eq. (5) and $v_{t}$ from Eq. (2). The resulting values of $v_{t} / v$ and $R e_{t}$ are summarized in Table 1 . It is seen that $\mathrm{v}_{t} / v$ progressively increases with increasing $R e$, reflecting the faster mixing rates at higher Reynolds numbers. The corresponding values of $R e_{t}$ exhibit a somewhat smaller rate of reduction as $R e$ increases.

Given $R e_{l}$, the measured radial profiles of streamwise mean velocities can be plotted according to the scaling of self-preserving laminar-like turbulent wakes from Eqs. (1) and (3). These results are plotted in Fig. 4, along with the predictions of Eqs. (1) and (3). The measurements are grouped into low, transition, and high Reynolds number regimes as before. All the measurements are within the laminar-like turbulent wake region with $x / d$ in the range 8-51. Measurements at larger $x / d$ are not shown in Fig. 4 because experimental accuracy was limited over an appreciable fraction of the wake cross-section due to the small mean velocities in this region. Results already discussed in connection with Fig. 3, however, have shown proper scaling of centerline velocities for $\left(x-x_{0}\right) / d>50$.

Although the measurements illustrated in Fig. 4 have been grouped into low, transition, and high Reynolds number regimes, results for all three regimes are essentially the same. For $\bar{u} / \bar{U}_{\infty}>$

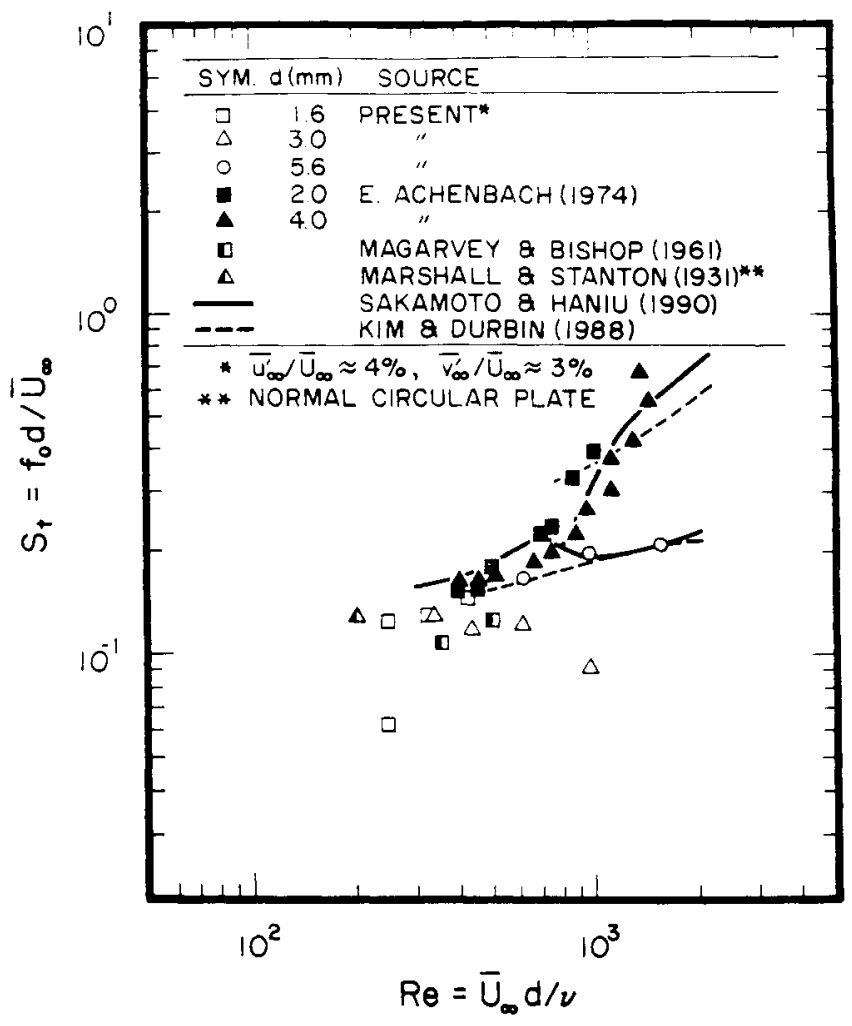

Fig. 2 Strouhal numbers of vortex shedding from the spheres. 
0.1 , the measurements agree with the predictions for self-preserving laminar-like turbulent wakes, Eqs. (1) and (3), within experimental uncertainties. Results near the edge of the flow exhibit a larger degree of scatter, which is attributed to larger experimental uncertainties of the velocity measurements in this region. Another factor limiting accuracy near the edge of the wakes is the approach of the sphere to the limits of the nearly constant ambient property region near the pipe axis. With laminar-like scaling for these wakes, the faster mixing at larger Reynolds numbers is reflected by increased values of the effective turbulent viscosity (see Table 1).

The value of the effective turbulent viscosity $v_{t}$ clearly is the critical parameter needed to estimate mean streamwise velocities in the laminar-like turbulent wake region. The results illustrated in Figs. 3 and 4 indicate that $v_{t}$ was relatively independent of position in the wake. However, $v_{t}$ varied in a rather systematic fashion with respect to the sphere Reynolds number. Results illustrating this behavior appear in Fig. 5, where $v_{t} / v$ is plotted as a function of $R e$ for all of the present measurements.

Similar to behavior observed in Fig. 3 for the near-wake region, $v_{t} / v$ in Fig. 5 exhibits distinct behavior in the low, transition, and high Reynolds number regimes. Over all three regimes, however, results for various sphere diameters overlap so that $v_{t} / v$ can be correlated as a function of $R e$ alone. This behavior suggests that length scale properties, like $\Lambda_{u} / d$ and $\ell_{K} / d$, have a relatively small effect on flow properties over the present test range, which is consistent with the apparently small variation of $v_{t} / v$ with position in the wake. The variation of $v_{t} / v$ with $R e$ is nearly linear in the low and high Reynolds number regimes and can be represented by the following empirical fits:

$$
\begin{array}{ll}
\mathrm{v}_{t} / \nu=0.017 R e, & 135<\operatorname{Re}<310 \\
\mathrm{v}_{t} / \nu=0.029 \operatorname{Re}, & 610<\operatorname{Re}<1560
\end{array}
$$

These results are only provisional for $\bar{u}_{\infty}^{\prime} / \bar{U}_{\infty}$ of about $4 \%$. In view of the vastly different behavior observed for spheres in nonturbulent environments ${ }^{3}$ and the present turbulent environments,

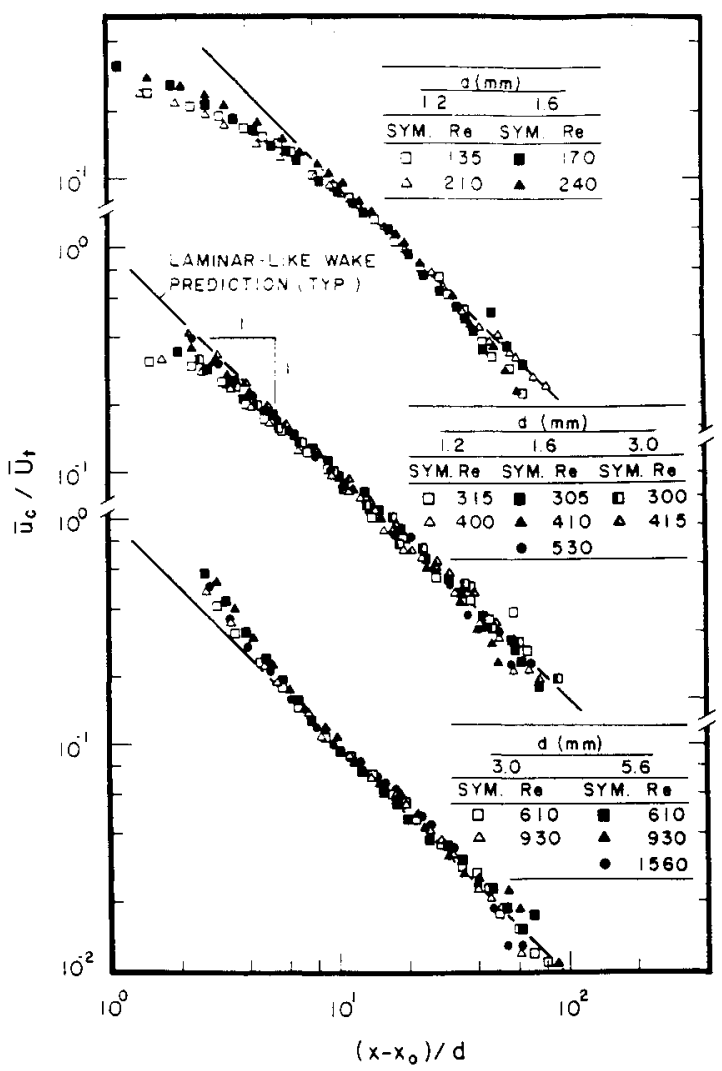

Fig. 3 Mean streamwise velocities along the axis of the wakes.

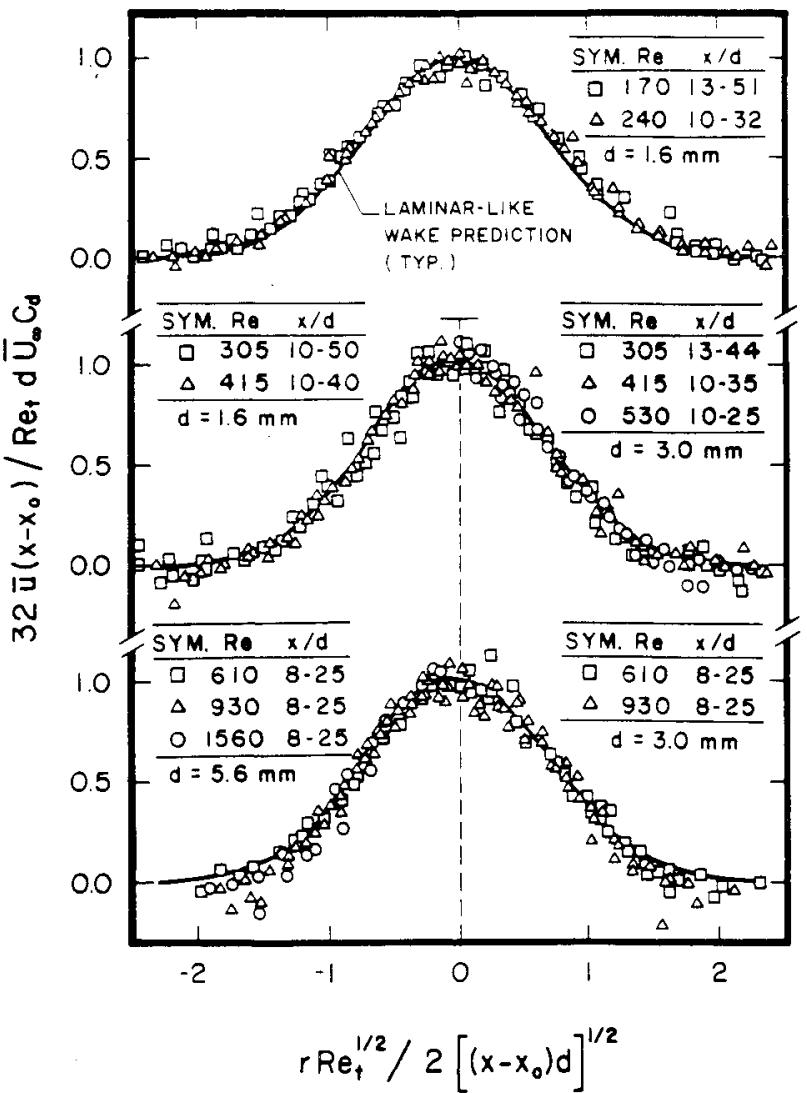

Fig. 4 Correlation of radial profiles of mean streamwise velocities in the laminar-like turbulent wake region.

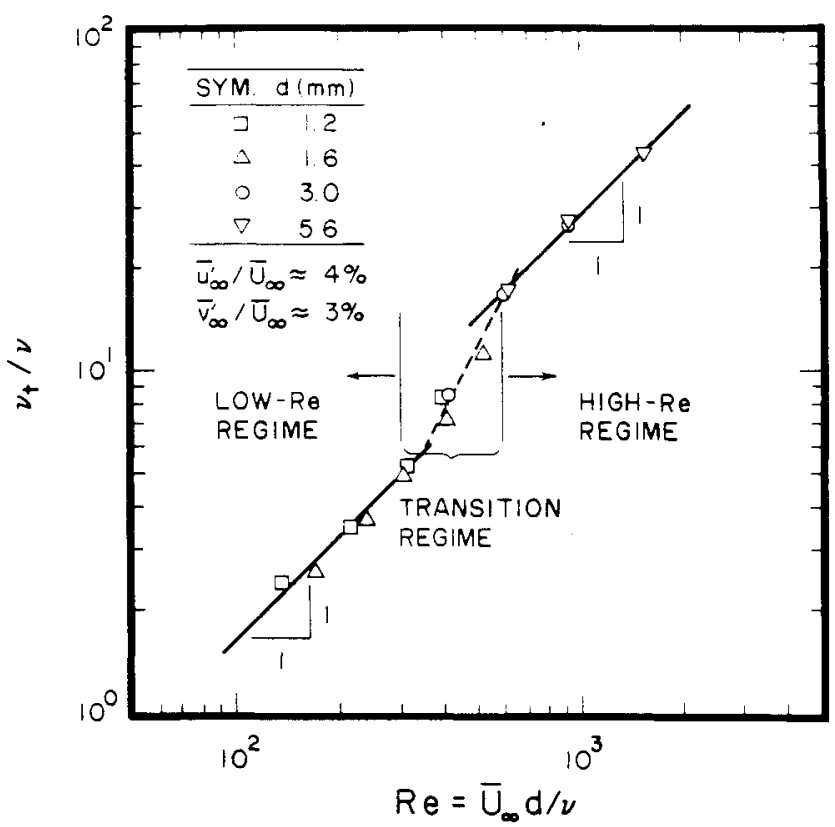

Fig. 5 Variation of turbulent viscosity with sphere Reynolds number.

effects of $\bar{u}_{\infty}^{\prime} / \bar{U}_{\infty}$ on $v_{t} / v$ certainly can be anticipated. Additionally, $v_{t} / N$ should approach unity as $R e$ becomes small so that Eq. (7) should not be used at $R e$ below the range of the present measurements. The rate of increase of $v_{t} / v$ with increasing $R e$ is more rapid in the transition regime than the other two regimes and can be approximated by the following empirical expression:

$$
v_{t} / \nu=1.2+42(\operatorname{Re} / 1000)^{2}, \quad 310<\operatorname{Re}<610
$$

The onset of the transition regime at $R e$ of about 300 corresponds to the onset of vortex shedding for present conditions, as noted ear- 


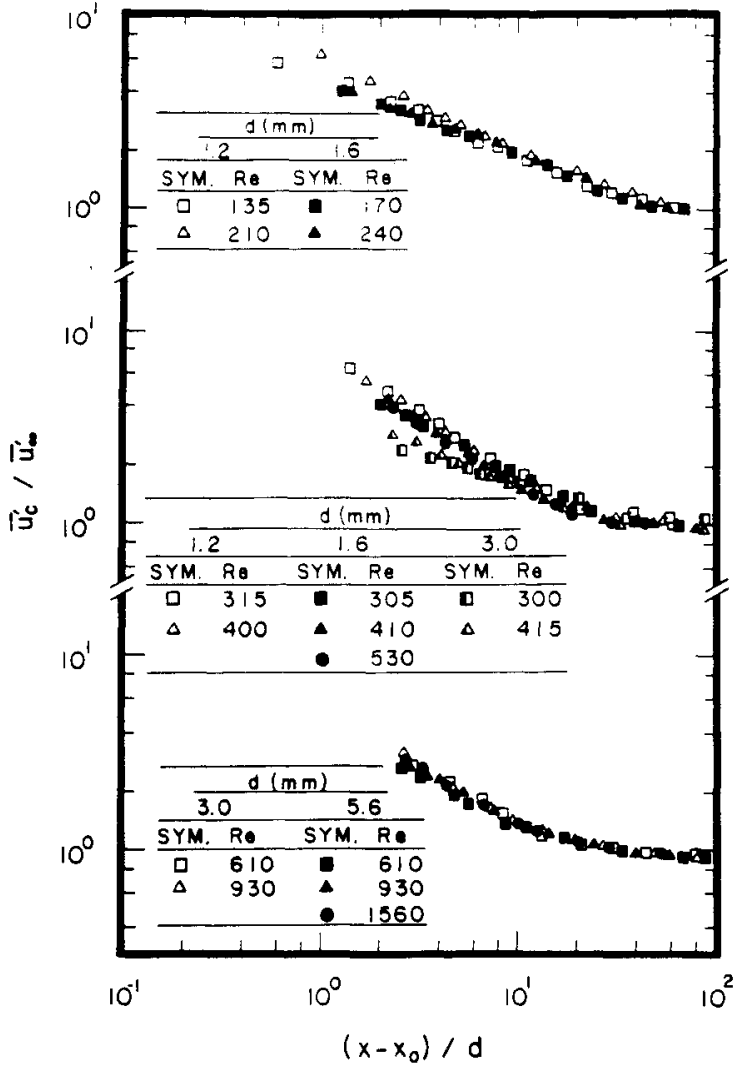

Fig. 6 Streamwise rms velocity fluctuations along the axis of the wakes.

lier. This suggests that vortex shedding is responsible for the more rapid growth of $v_{t} / v$ with $R e$ in the transition regime, with this growth period ending when the near-wake region becomes fully developed in the presence of vortex shedding, much like the nearwake mean velocity profiles illustrated in Fig. 3. Measured velocity fluctuations in the wakes tend to support this behavior and will be considered next.

\section{Velocity Functions}

Streamwise and cross-stream velocity fluctuations along the wake axis are plotted as functions of $\left(x-x_{0}\right) / d$ in Figs. 6 and 7. The velocity fluctuations have been normalized by the corresponding ambient rms velocity fluctuations, to indicate the degree of enhancement of turbulence levels within the wake. As before, the measurements have been grouped into low, transition, and high Reynolds number regimes. Aside from a few measurements in the near-wake region, the results are in the laminar-like turbulent wake region with $\left(x-x_{o}\right) / d$ in the range $8-100$.

The streamwise velocity fluctuations, Fig. 6 , are relatively independent of $R e$ when normalized by $\bar{u}_{\infty}^{\prime}$. In addition, results in all three Reynolds number regimes are essentially the same, aside from a somewhat increased degree of scatter in the near-wake region for the transition Reynolds number regime. Streamwise velocity fluctuations exhibit significant enhancement in the near wake, becoming 4-6 times greater than ambient turbulence levels. However, streamwise velocity fluctuations decrease monotonically with increasing streamwise distance and approach ambient turbulence levels at $\left(x-x_{o}\right) / d \approx 60$, which corresponds to the region where mean wake velocities at the axis approach ambient velocity fluctuation levels (cf. Fig. 3).

The behavior of $\bar{v}_{c}^{\prime} / \bar{v}_{\infty}^{\prime}$ in Fig. 7 differs from $\bar{u}_{c}^{\prime} / \bar{u}_{\infty}^{\prime}$ in Fig. 6 . First of all, $\bar{v}_{c}^{\prime} / \bar{v}_{\infty}^{\prime}$ tends to vary with $R e$ to a greater degree, even within each of the three Reynolds number regimes. The exception is a tendency to approach behavior independent of Reynolds number for $R e>9 \times 10^{2}$, where $\bar{v}_{c}^{\prime} \approx \bar{u}_{c}^{\prime}$ and $\bar{v}_{c}^{\prime} / \bar{v}_{\infty}^{\prime}$ reaches its largest values at each streamwise position. In the low Reynolds number regime, there is a region in the near wake where the presence of the sphere reduces the cross-stream velocity fluctuations slightly from

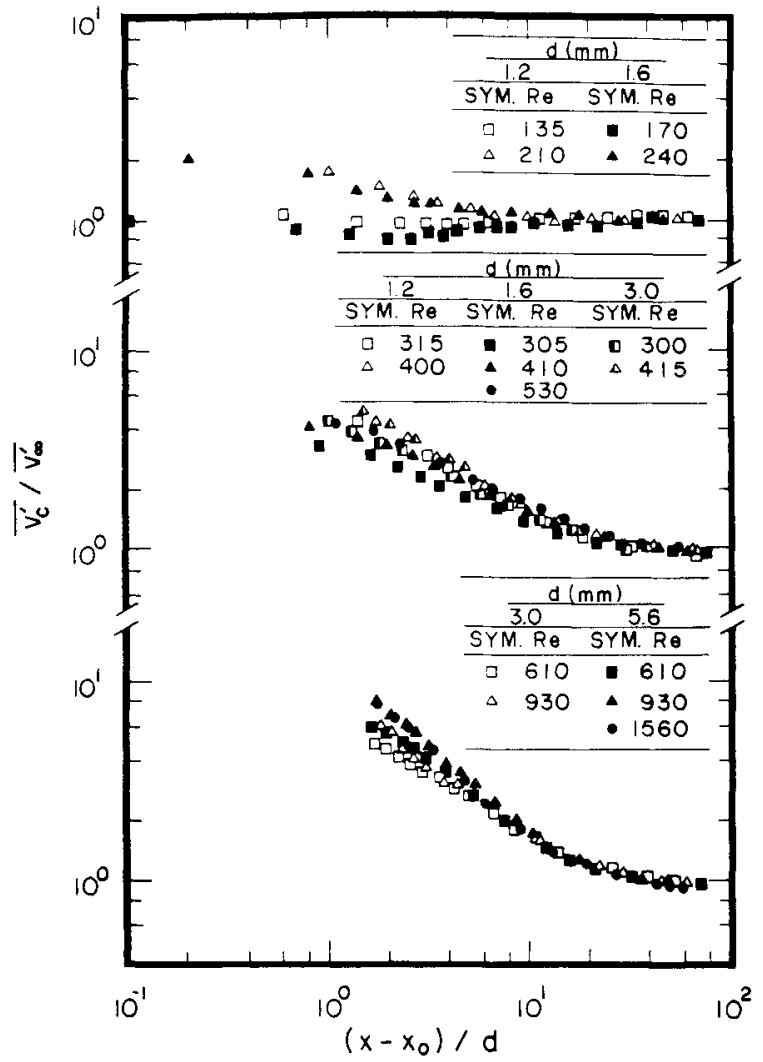

Fig. 7 Cross-stream rms velocity fluctuations along the axis of the wakes.

ambient values. This behavior probably involves effects of the recirculation zone behind the spheres, ${ }^{3-9}$ however, the mechanism causing velocity fluctuations to be damped was not resolved. The onset of vortex shedding as the transition region is reached causes $\bar{v}_{c}^{\prime} / \bar{v}_{\infty}^{\prime}$ to increase substantially in the near-wake region. Subsequently, cross-stream velocity fluctuations progressively increase with increasing $R e$ until they stabilize at $R e>9 \times 10^{2}$, as discussed earlier.

The results of Figs. 6 and 7 indicate that turbulence levels within laminar-like turbulent wakes are substantially enhanced from ambient turbulence levels; thus, it is not surprising that $v_{t} / v$ tends to vary with $R e$ even when $\bar{u}_{\infty}^{\prime} / \bar{U}_{\infty}$ is a constant. Furthermore, the relatively small values of $\bar{v}_{c}^{\prime} / \bar{v}_{\infty}^{\prime}$, approaching unity at the lowest Reynolds numbers, are consistent with relatively small effects of turbulence so that $v_{t} / v$ approaches unity as well. Additionally, although, $\bar{v}_{c}^{\prime} / \bar{v}_{\infty}^{\prime}$ tends to increase with $R e$ over the test range, the onset of vortex shedding initiates conditions where $\bar{v}_{c}^{\prime} / \bar{v}_{\infty}^{\prime}$ increases most rapidly, which also is consistent with the more rapid increase of $v_{t} / v$ with $R e$ in this region (cf. Fig. 5). Furthermore, it seems plausible that the period of unusually rapid growth of $v_{t} / v$ should end when values of $\bar{u}_{c}^{\prime} / \bar{u}_{\infty}^{\prime}$ and $\bar{v}_{c}^{\prime} / \bar{v}_{\infty}^{\prime}$ become relatively independent of $R e$ at high Reynolds numbers. Finally, values of $\bar{u}_{c}^{\prime} / \bar{u}_{\infty}^{\prime}$ and $\bar{v}_{c}^{\prime} / \bar{v}_{\infty}^{\prime}$ for different sphere diameters are essentially the same at given values of $R e$ and $\left(x-x_{0}\right) / d$, supporting the idea that variations of $\Lambda_{u} / d$ and $\ell_{K} / d$ have a relatively small effect on wake turbulence properties, and thus $v_{t} / \nu$, over the present test range.

\section{Conclusions}

The structure of the wakes of spheres in a turbulent environment was observed for $R e=135$ to 1560 . The turbulent environment involved conditions near the axis of fully developed turbulent pipe flow with turbulence intensities of roughly $4 \%, \Lambda_{u} / d$ in the range $11-59$, and $\ell_{K} / d$ in the range $0.08-0.80$. The major conclusions of the study are as follows:

1) The wakes were turbulent, but mean streamwise velocities scaled like self-preserving laminar wakes with enhanced viscosities due to the effects of turbulence. 
2) The effective turbulent viscosities were independent of position in the wake and the length scale but increased with increasing $R e$, yielding $v_{t} / v$ in the range $2.4-44.3$. The $v_{t} / v$ exhibited low and high $R e$ regimes, with transition between these regimes for $R e$ in the range 300 to 610 where the rate of increase of $v_{t} / v$ with increasing $R e$ was more rapid.

3) Streamwise and cross-stream velocity fluctuations along the wake axis reflected the different properties of the three sphere Reynolds number regimes, with behavior relatively independent of $R e$ in the low and high $R e$ regimes, whereas effects of $R e$ were observed in the transition regime. Turbulence intensities in the nearwake region were lowest and highest in the low and high Re regimes, respectively, and exhibited small effects of the length scale ratios, $\Lambda_{u} / d$ and $\ell_{K} / d$, over the present test range.

4) The onset of the transition regime corresponded to the onset of vortex shedding from the spheres ( $R e$ of about 300 ) whereas the transition regime ended when effects of vortex shedding no longer could be observed in the temporal power spectra of cross-stream velocity fluctuations in the near-wake region (at the axis for $x / d=$ 4). Thus, the enhanced rates of turbulent mixing in the high $R e$ regime appear to be associated with the development of effects of vortex shedding in the near-wake region.

5) The onset of vortex shedding at $R e$ of about 300 , the values of $S t$ associated with vortex-shedding frequencies, and the drag coefficients of the spheres were similar to observations for spheres in nonturbulent environments. This behavior is plausible due to the relatively large values of $\Lambda_{u} / d$ of present tests so that the largescale motions approached quasisteady behavior.

Effects of varying ambient turbulent intensities must still be resolved in order to address the properties of turbulence generation in dispersed multiphase flows like sprays. ${ }^{1,2}$ Additionally, a broader range of $\Lambda_{u} / d$ and $\ell_{K} / d$ should be investigated in order to resolve effects of ambient turbulence scales that are likely to affect vortex shedding and the mixing properties of wakes in some turbulent environments.

\section{Acknowledgments}

This research was sponsored by the Air Force Office of Scientific Research, Air Force Systems Command, U.S. Air Force, under Grants AFOSR-89-0516 and F49620-92-J-0399. The U.S. Government is authorized to reproduce and distribute copies for government purposes, notwithstanding any copyright thereon.

\section{References}

'Parthasarathy, R. N., and Faeth, G. M., "Turbulence Modulation in Homogeneous Dilute Particle-Laden Flows," Journal of Fluid Mechanics, Vol. 220, Pt. 2, 1990, pp. 485-514.

${ }^{2}$ Mizukami, M., Parthasarathy, R. N., and Faeth, G. M., "Particle-Generated Turbulence in Homogeneous Dilute Dispersed Flows," International Journal of Multiphase Flow, Vol. 18, No. 3, 1992, pp. 397-412.

${ }^{3} \mathrm{Wu}$, J.-S., and Faeth, G. M., "Sphere Wakes in Still Surroundings at Intermediate Reynolds Numbers," AIAA Journal, Vol. 31, No. 8, 1993, pp. 1448-1455.

${ }^{4}$ Margarvey, R. H., and Bishop, R. L., "Wakes in Liquid-Liquid Systems," Physics of Fluids, Vol. 4, No. 7, 1961, pp. 800-805.

${ }^{5}$ Magarvey, R. H., and MacLatchy, C. S., "Vortices in Sphere Wakes," Canadian Journal of Physics, Vol. 43, Sept. 1965, pp. 1649-1656.

${ }^{6}$ Goldberg, A., and Florsheim, B. H., "Transition and Strouhal Number for the Incompressible Wake of Various Bodies," Physics of Fluids, Vol. 9 , No. 1, 1966, pp. 45-50.

${ }^{7}$ List, R., and Hand, M. J., "Wakes of Freely Falling Water Drops," Physics of Fluids, Vol. 14, No. 8, 1971, pp. 1648-1655.
${ }^{8}$ Achenbach, E., "Vortex Shedding from Spheres," Journal of Fluid Mechanics, Vol. 62, Pt. 2, 1974, pp. 209-221.

${ }^{9}$ Nakamura, I., "Steady Wake Behind a Sphere," Physics of Fluids, Vol. 19, No. 1, 1976, pp. 5-8.

${ }^{10} \mathrm{Pao}$, H.-P., and Kao, T. K., "Vortex Structure in the Wake of a Sphere," Physics of Fluids, Vol. 20, No. 2, 1977, pp. 187-191.

${ }^{11}$ Sakamoto, H., and Haniu, H., "A Study on Vortex Shedding from Spheres in a Uniform Flow," Journal of Fluids Engineering, Vol. 112, No. 4, 1990, pp. 386-392.

${ }^{12}$ Kim, K. J., and Durbin, P. A., "Observation of the Frequencies in a Sphere Wake and Drag Increase by Acoustic Excitation," Physics of Fluids, Vol. 31, No. 11, 1988, pp. 3260-3265.

${ }^{13}$ Carmody, T., "Establishment of the Wake Behind a Disk," Journal of Basic Engineering, Vol. 87, No. 4, 1964, pp. 869-883.

${ }^{14} \mathrm{Hwang}$, N. H. C., and Baldwin, L. V., "Decay of Turbulence in Axisymmetric Wakes," Journal of Basic Engineering, Vol. 88, No. 1, 1966, pp. 261-268.

${ }^{15}$ Chevray, R., "The Turbulent Wake of a Body of Revolution," Journal of Basic Engineering, Vol. 90, No. 2, 1968, pp. 275-284.

${ }^{16}$ Uberoi, M. S., and Freymuth, P., "Turbulent Energy Balance and Spectra of the Axisymmetric Wake," Physics of Fluids, Vol. 13, No. 9, 1970, pp. 2205-2210.

${ }^{17}$ Tennekes, H., and Lumley, J. L., A First Course in Turbulence, MIT Press, Cambridge, MA, 1972, pp. 113-124.

${ }^{18}$ Hinze, J. O., Turbulence, 2nd ed., McGraw-Hill, New York, 1975, pp. 496-581.

${ }^{19}$ Schlichting, H., Boundary Layer Theory, 7th ed., McGraw-Hill, New York, 1977, pp. 234, 235, 599

${ }^{20}$ Phillips, O. M., "The Final Period of Decay of Non-Homogeneous Turbulence," Proceedings of the Cambridge Philosophical Society, Vol. 52, Pt. I, 1956, pp. 135-151.

${ }^{21}$ Lee, D. A., and Tan, H. S., "Study of Inhomogeneous Turbulence," Physics of Fluids, Vol. 10, No. 6, 1967, pp. 1224-1230.

${ }^{22}$ Torobin, L. B., and Gauvin, W. H., "Fundamenta 1 Aspects of SolidsGas Flow, Part V: The Effects of Fluid Turbulence on the Particle Drag Coefficient," Canadian Journal of Chemical Engineering, Vol. 38, No. 6, 1960, pp. 189-200.

${ }^{23}$ Komoda. H., "On the Effect of Free-Stream Turbulence on the Structure of Turbulent Wake," Journal of the Japan Society of Aeronautical Engineering. Vol. 5, Oct. 1957 , pp. 274-279.

${ }^{24} \mathrm{Pal}$, S., and Raj, R., "Wake Behavior in the Presence of Free Stream Turbulence," Journal of Engineering for Power, Vol. 103, No. 3, 1981, pp. $490-498$

${ }^{25} \mathrm{Pal}, \mathrm{S}$., "Freestream Turbulence Effects on Wake Properties of a Flat Plate at an Incidence," AIAA Journal, Vol. 23, No. 12, 1985, pp. 18681871.

${ }^{26}$ Faeth, G. M., "Mixing, Transport and Combustion in Sprays," Progress in Energy and Combustion Science, Vol. 13, No. 4, 1987, pp. 293-345.

${ }^{27}$ Kürten, H., Raasch, J., and Rumpf, H., "Beschleunigung eines Kugelförmen Feststoffteilchens im Strömungsfeld Konstanter Geschwindigkeit," Chemie-Ingenieur-Technik, Vol. 38, No. 9, 1966, pp. 941-948.

${ }^{28}$ Laufer, J., "The Structure of Turbulence in Fully Developed Pipe Flow," NACA Rept. 1174, Aug. 1954.

${ }^{29}$ Wu, M.-S., Kwon, S., Driscoll, J. F., and Faeth, G. M., "Preferential Diffusion Effects on the Surface Structure of Turbulent Premixed Hydrogen/Air Flames," Combustion Science and Technology, Vol. 78, Sept. 1991, pp. 69-96.

${ }^{30}$ Ruff, G. A., "Structure and Mixing Properties of the Near-Injector Region of Nonevaporating Pressure-Atomized Sprays," Ph.D. Thesis, Dept. of Aerospace Engineering, Univ. of Michigan, Ann Arbor, MI, 1990, pp. $54-58$.

${ }^{31}$ Durão, D. F. G., Heitor, M. V., and Pereira, J. C. F., "Measurements of Turbulent and Periodic Flows Around a Square Cross-Section Cylinder," Experiments in Fluids, Vol. 6, No. 4, 1988, pp. 298-304.

${ }^{32}$ Marshall, D., and Stanton, T. E., "On the Eddy System in the Wake of Flat Circular Plates in Three-Dimensional Flow," Proceedings of the Royal Society of London, Vol. A130, No. A813, 1931, pp. 295-301. 